\title{
Is bottomonium suppression in proton-nucleus and nucleus-nucleus collisions at LHC energies due to the same effects?
}

\author{
E.G. Ferreiro ${ }^{a, b}$ and J.P. Lansberg ${ }^{c}$ \\ ${ }^{a}$ Laboratoire Leprince-Ringuet, Ecole polytechnique, CNRS/IN2P3, Université Paris-Saclay, \\ Palaiseau, F-91128 France \\ ${ }^{b}$ Departamento de Física de Partículas and IGFAE, Universidade de Santiago de Compostela, \\ Santiago de Compostela, 15782 Spain \\ ${ }^{c}$ IPNO, Université Paris-Saclay, Université Paris-Sud, CNRS/IN2P3, \\ Orsay, F-91406 France \\ E-mail: elena@fpaxp1.usc.es, Jean-Philippe.Lansberg@in2p3.fr
}

ABSTRACT: We show that we can reproduce all the features of the bottomonium suppression in both proton-nucleus and nucleus-nucleus collisions at LHC energies in a comoverinteraction picture. For each collision system, we use the measured relative suppression of the excited $\Upsilon(2 S)$ and $\Upsilon(3 S)$ states to $\Upsilon(1 S)$ by ATLAS and CMS to parametrise the scattering cross sections of all $S$ - and $P$-wave bottomonia with the comoving particles created during the collisions. In addition to a single nonperturbative parameter, these cross sections depend on the momentum distribution of these comovers which we found to be the same for proton-nucleus and nucleus-nucleus collisions as well as for partonic and hadronic comovers. Moreover, we can also reproduce the absolute suppresion rates measured by ALICE, ATLAS, CMS and LHCb when the nuclear modifications of the parton densities are taken into account.

Keywords: Heavy Ion Phenomenology, Phenomenological Models

ARXIV EPRINT: 1804.04474 


\section{Contents}

1 Introduction 1

2 The comover interaction model $\quad 3$

$\begin{array}{lll}3 & \text { Fitting the data } & 6\end{array}$

4 Relative NMFs $\quad 8$

5 Absolute NMFs $\quad 9$

6 Conclusions 10

\section{Introduction}

By the end of the year 2013, following the LHC $p \mathrm{~Pb}$ run at $\sqrt{s_{N N}}=5 \mathrm{TeV}$, the CMS collaboration probably reported the most unexpected observation of the LHC heavy-ion programme: the excited $\Upsilon(2 S)$ and $\Upsilon(3 S)$ states were experiencing more suppression than the lower $\Upsilon(1 S)$ state [1]. At low collision energies, such a relative suppression would naturally follow from final-state interactions with the remnants of the colliding lead nucleus since the excited states have a larger size than the ground state.

However, for $p \mathrm{~Pb}$ collisions at the LHC, the quarkonium-formation time in the beamnucleus rest frame is expected to be larger than the nucleus radius because of the large rapidity difference ${ }^{1}$ between the nucleus beam and the produced $b \bar{b}$ pair. As such, it has not evolved into any physical $n S$ or $n P$ state when it passes through the nuclear matter contained in the nucleus. Consequently, one cannot invoke the interaction with the nucleon in the nucleus to explain the relative suppression observed by CMS, neither can one invoke initial-state effects such as the modification of parton distribution functions (PDFs) [2-6] or coherent energy loss [7], which are known to have a similar impact on the different bottomonia [8]. Very recently, the ATLAS collaboration confirmed [9] the observation of these relative suppressions with very similar magnitudes.

Not only was this result totally unforeseen, but it casted serious doubts on the conventional interpretation of the relative suppression of bottomonium earlier observed by CMS in lead-lead collisions [10, 11]: the excited $\Upsilon(2 S)$ and $\Upsilon(3 S)$ states are larger, less tightly bound and thus suffer more from the colour screening and related effects in the quark-gluon plasma (QGP).

Assuming that the phenomena responsible for the suppression observed in a $p \mathrm{~Pb}$ collision remains similar in a $\mathrm{PbPb}$ collision, one is entitled to factorise the effects coming

\footnotetext{
${ }^{1}$ Always above 4 .
} 
from each $\mathrm{Pb}$ nucleus and thus, at central rapidities, to square the measured suppression factor [12] in $p \mathrm{~Pb}$ collisions to extrapolate to $\mathrm{PbPb}$ collisions. This is, for instance, what is done if one assumes that such a suppression comes from the nuclear modification of PDFs $[8,13-18]$. Although it does not rely on any theorem, it remains the most realistic assumption if one considers that these effects are not enhanced in a nucleus-nucleus collision. By the same token, one can extrapolate the relative suppression factor ${ }^{2}$ for $\mathrm{PbPb}$ collisions by squaring the $p \mathrm{~Pb}$ ones. As such, one respectively obtains

$$
R_{\mathrm{PbPb} \text { from pPb }}^{\Upsilon(2 S) /(1 S)} \simeq 0.65 \quad \text { and } \quad R_{\mathrm{PbPb} \text { from pPb }}^{\Upsilon(3 S) \Upsilon 0.5}
$$

to be compared with the experimental ratios reported by $\mathrm{CMS}$ in $\mathrm{PbPb}$ collisions at $5.02 \mathrm{TeV}[19]$, namely

$$
R_{\mathrm{PbPb}}^{\Upsilon(2 S) / \Upsilon(1 S)} \simeq 0.3 \quad \text { and } \quad R_{\mathrm{PbPb}}^{\Upsilon(3 S) / \Upsilon(1 S)} \text { compatible with } 0 .
$$

Clearly the extrapolated $p \mathrm{~Pb}$ effects are significant and need to be understood for a proper interpretation of the $\mathrm{PbPb}$ results.

In this work, we attempt to explain these relative suppression in $p \mathrm{~Pb}$ and $\mathrm{PbPb}$ collisions altogether by assuming that the bottomonia are broken by collisions with comoving particles - i.e. particles with similar rapidities - and whose density is directly connected to the particle-multiplicity measured at that rapidity for the corresponding colliding system.

In such a scenario, an increase in the colliding energy has the opposite effect than for the suppression by the nucleus remnants. Instead of decreasing because of color transparency, or because the propagating pair can only interact with the remnants in the very early phase of its formation, the suppression by these comovers increases because the number of the produced particles from a given $p \mathrm{~Pb}$ collision increases with energy. So does the number of comoving particles along with the heavy-quark pair. Another important feature of this assumption is that the heavy-quark pairs have reached - in their rest frame - a physical state after a fraction of a femtometer. The $\Upsilon(1 S), \Upsilon(2 S)$ and $\Upsilon(3 S)$ states then interact with very different probabilities with these comoving particles, which provides a very natural explanation for the observed relative suppression.

As aforementioned, nobody expected this relative suppression in $p \mathrm{~Pb}$ collisions and, as for now, no other effect have been proposed to explain it apart from suggesting the creation of a "hot" medium in these high-energy proton-nucleus $(p A)$ collisions. None of the known "cold" nuclear-matter effects generate a relative suppresion. It is thus in fact the ideal observable to fix the comover-bottomonium cross sections, which are the only new phenomenological quantities entering our study. Surprisingly, the CIM has never been applied to the bottomonia.

We in fact go one step further by proposing an improved version of the well-established comover interaction model (CIM) [20-27], already successfully applied to explain a similar

\footnotetext{
${ }^{2}$ For the record, the (absolute) nuclear modification factor (NMF) for $A B$ collisions is defined as $R_{A B}^{\Upsilon(n S)}=d N_{A B}^{\Upsilon(n S)} /\left(\left\langle N_{\text {coll }}\right\rangle d N_{p p}^{\Upsilon(n S)}\right.$ where $\left.d N_{A B(p p)}^{\Upsilon}(n S)\right)$ is the $\Upsilon(n S)$ yield per $A B(p p)$ collisions (in the corresponding centrality class) and $\left\langle N_{\text {coll }}\right\rangle$ is the average number of binary nucleon-nucleon collisions per $A B$ collisions (in the same centrality class). Beside, the relative nuclear-modification factors (or double ratios) are simply their ratios such that $R_{A B}^{\Upsilon(m S) / \Upsilon(n S)} \equiv R_{A B}^{\Upsilon(m S)} / R_{A B}^{\Upsilon(n S)}$.
} 
unexpected suppression of excited charmonia [28]. Indeed, instead of independently fixing the cross sections state by state, we propose a generic formula for all the quarkonium states and suggest a connection with the momentum (or energy) distribution of the comovers in the transverse plane, thus with an effective temperature $\left(T_{\text {eff }}\right)$ of the comovers. With such an approach, we are able to propose a clear benchmark between $p A$ and $A A$ collisions under the CIM paradigm.

As we shall see, the approach is particularly successful:

(i) the interaction strengths between the bottomonia and the comovers needed to reproduce the $p \mathrm{~Pb}$ data follow a simple pattern in terms of the size and the binding energy - both calculable with a simple Schrödinger equation - of all the bottomonium states, which renders our set-up predictive;

(ii) even more striking, the entire relative suppression observed in $\mathrm{PbPb}$ collisions is accounted for by scatterings with comovers with remarkably similar interaction strengths as for the $p \mathrm{~Pb}$ data;

(iii) the absolute magnitude of the $\Upsilon$ suppression in $p \mathrm{~Pb}$ and $\mathrm{PbPb}$ collisions is also well reproduced up to the uncertainties in the nuclear modification of PDFs.

Overall, as we will show, all the LHC $p \mathrm{~Pb}$ and $\mathrm{PbPb}$ data can be reproduced with merely two parameters.

\section{The comover interaction model}

Within this model, the quarkonia are suppressed by the interaction with the comoving medium, constituted by particles with similar rapidities. At a time $\tau$, the rate equation that governs the density of quarkonium at a given transverse coordinate $s$ and rapidity $y$ for a collision of impact parameter $b, \rho^{\Upsilon}(b, s, y)$, obeys the expression

$$
\tau \frac{\mathrm{d} \rho^{\Upsilon}}{\mathrm{d} \tau}(b, s, y)=-\left\langle\sigma^{\mathrm{co}-\Upsilon}\right\rangle \times \rho^{\mathrm{co}}(b, s, y) \times \rho^{\Upsilon}(b, s, y),
$$

where $\left\langle\sigma^{\text {co- }} \Upsilon\right\rangle$ is the (energy averaged) cross section of bottomonium dissociation due to interactions with the comoving medium characterised by the transverse density $\rho^{\mathrm{co}}(b, s, y)$ at $\tau_{i}$. We consider the medium to be Bjorken-like and the dilution of the comover densities as a function of time due to a longitudinal expansion is taken into account. On the contrary, we neglect any possible dilution in the transverse plane. By integrating this equation from $\tau_{i}$ to $\tau_{f}$, one obtains the survival probability $S_{\Upsilon}^{\text {co }}(b, s, y)$ of a $\Upsilon$ interacting with comovers:

$$
S_{\Upsilon}^{\mathrm{co}}(b, s, y)=\exp \left\{-\left\langle\sigma^{\mathrm{co}-\Upsilon}\right\rangle \times \rho^{\mathrm{co}}(b, s, y) \times \ln \left(\frac{\rho^{\mathrm{co}}(b, s, y)}{\rho_{p p}^{\mathrm{co}}(y)}\right)\right\} .
$$

The argument of the logarithm comes from $\tau_{f} / \tau_{i}$ converted in ratios of comover densities assuming that the interaction stops when the comover density has diluted down to that reached in a $p p$ collision at the same energy and rapidity $\left(\rho_{p p}^{\mathrm{co}}(y)\right)$. 
When computing $S_{\Upsilon}^{\mathrm{co}}(b, s, y)$, the density of comovers $\rho^{\mathrm{co}}$ is considered as a controlled input which we assume to satisfy the following condition:

$$
\rho^{\mathrm{co}}(b, s, y)=\mathcal{F}_{\text {shadowing }}^{\mathrm{co}}(b, s) \times \frac{3}{2} \frac{d N_{\mathrm{ch}}^{p p}}{d y} \times \frac{d N_{\mathrm{coll}}(b)}{d^{2} s},
$$

where

(i) $\mathcal{F}_{\text {shadowing }}^{\text {co }}$ is a suppression factor accounting for the shadowing of the parton flux in a nucleus affecting the charged-particle multiplicity, and thus that of the comovers; it should not be confused with the shadowing factor applicable to hard scatterings;

(ii) $\frac{d N_{\mathrm{ch}}^{p p}}{d y}$ is the (measured) rapidity-differential charged-particle multiplicity in $p p$ collisions;

(iii) the factor $3 / 2$ accounts for the neutral comovers;

(iv) $N_{\text {coll }}(b)$ is the number of binary nucleon-nucleon collisions at a given impact parameter $b$. It is computed with a Glauber-model Monte Carlo.

For the parametrisation of $\mathcal{F}_{\text {shadowing }}^{\text {co }}$ used in ref. [29], a good description of the centrality dependence of charged multiplicities in nuclear collisions is obtained both at RHIC and LHC energies. We thefore adopt it here as well.

For $p A$ collisions, it is most natural to take the medium as made of pions. Nevertheless, we will show later that the nature of this medium - partonic or hadronic - does not qualitatively change our results which is one of the important findings of our study.

As can be seen from eq. (2.1), the main ingredient driving the abundance of a given bottomonium is its interaction cross section with the comovers, $\left\langle\sigma^{\mathrm{co}-} \Upsilon\right\rangle$. In our previous works on charmonia, these were obtained from fits to low-energy $A A$ data $[21],\left\langle\sigma^{\mathrm{co}-J / \psi}\right\rangle=$ $0.65 \mathrm{mb}$ and $\left\langle\sigma^{\mathrm{co}-\psi(2 S)}\right\rangle=6 \mathrm{mb}$. Such - purely phenomenological - cross sections in fact would result from the convolution of the comover-energy distribution in the transverse plane and the energy-dependent comover-quarkonium cross section. As such they may slightly depend on the collision energy via a change of the comover-energy distribution. Yet, these values were successfully applied at higher energies to reproduce [28] $J / \psi$ and $\psi(2 \mathrm{~S}) p A$ data at RHIC and the LHC as well as $A A$ data accounting for the recombination of charm quarks [26, 27].

One can not follow the same approach for $\Upsilon(n S)$ since no $A A$ relative-suppression bottomonium data exist at low energies and, in fact, the CIM was never applied to bottomonia before. In addition, the bottomonium family is richer with at least 6 phenomelogical cross sections to be considered in a full computation. We have thus adopted another strategy by going to a slightly more microscopic level accounting for the energy distribution of the comover-quarkonium cross section and that of the comovers in the transverse plane. This in fact allowed us to reduce the degrees of freedom of our modeling to the introduction of essentially 2 parameters, yet applicable to the entire bottomonium family and allowing us to investigate the nature the comovers (gluons or pions). 
To do so we assumed that:

(i) the thresholds, $E_{\mathrm{thr}}^{\mathcal{Q}}$, approximately follow from the mass differences between the quarkonium, $\mathcal{Q}$, and the lightest open beauty hadron pair, taking into account the comover mass;

(ii) away from the thresholds, the cross section should scale like the geometrical cross section, $\sigma_{\text {geo }}^{\mathcal{Q}} \simeq \pi r_{\mathcal{Q}}^{2}$, where $r_{\mathcal{Q}}$ is the quarkonium Bohr radius. It can be evaluated by solving the Schrödinger equation with a well-choosen potential reproducing the quarkonium spectroscopy [30].

Our parametrisation of the energy dependence thus simply amounts to interpolating from $\sigma^{\mathrm{co}-\mathcal{Q}}\left(E^{\mathrm{co}}=E_{\mathrm{thr}}^{\mathcal{Q}}\right)=0$ at threshold up to $\sigma^{\mathrm{co}-\mathcal{Q}}\left(E^{\mathrm{co}} \gg E_{\mathrm{thr}}^{\mathcal{Q}}\right)=\sigma_{\text {geo }}^{\mathcal{Q}}$ away from threshold but with the same dependence for all the states. It reads

$$
\sigma^{\mathrm{co}-\mathcal{Q}}\left(E^{\mathrm{co}}\right)=\sigma_{\text {geo }}^{\mathcal{Q}} \times\left(1-\frac{E_{\mathrm{thr}}^{\mathcal{Q}}}{E^{\mathrm{co}}}\right)^{n}
$$

where $E_{\mathrm{thr}}^{\mathcal{Q}}=M_{\mathcal{Q}}+m_{\mathrm{co}}-2 M_{B}$ is the threshold energy to break the quarkonium bound state and $E^{\mathrm{co}}=\sqrt{p^{2}+m_{\mathrm{co}}^{2}}$ is the energy of the comover in the quarkonium rest frame. In the case of a hadronic medium (made of pions), $m_{\mathrm{co}}=0.140 \mathrm{GeV}$, while it is zero for gluons. The geometrical cross sections $\sigma_{\text {geo }}^{\mathcal{Q}}$ which we used are shown in table 1 , together with the threshold energies $E_{\mathrm{thr}}^{\mathcal{Q}}$ and the bottomonium radii. The first free parameter of our modeling, $n$, characterises how quickly the cross section approaches the geometrical cross section. Attempts to compute this energy dependence, using the multipole expansion in perturbative QCD at LO [30-32], would suggest that $n$ is close to 4 for pion comovers by making the strong assumption that the scattering is initiated by gluons inside these pions. Hadronic models which take into account non-perturbative effects and thus most likely provide a better description of the physics at work [33] show a different energy dependence. It effectively corresponds to smaller $n$ [34]. As such, we will consider $n$ varying from 0.5 to 2 . In fact, the discrepancies existing between the aforementioned LO QCD results and these hadronic calculations are partly due to large higher order correction near the threshold [35].

As for the energy distribution of the comovers in the transverse plane, we simply take a Bose-Einstein distribution

$$
\mathcal{P}\left(E^{\mathrm{co}} ; T_{\text {eff }}\right) \propto \frac{1}{e^{E^{\mathrm{co}} / T_{\text {eff }}}-1}
$$

which introduces our second parameters, namely an effective temperature of these comovers.

Having $\mathcal{P}\left(E^{\mathrm{co}} ; T_{\text {eff }}\right)$ and $\sigma^{\mathrm{co}-\mathcal{Q}}\left(E^{\mathrm{co}}\right)$, we derive the energy-averaged quarkoniumcomover-interaction cross section

$$
\left\langle\sigma^{\mathrm{co}-\mathcal{Q}}\right\rangle\left(T_{\mathrm{eff}}, n\right)=\frac{\int_{0}^{\infty} d E^{\mathrm{co}} \mathcal{P}\left(E^{\mathrm{co}} ; T_{\mathrm{eff}}\right) \sigma^{\mathrm{co}-\mathcal{Q}}\left(E^{\mathrm{co}}\right)}{\int_{0}^{\infty} d E^{\mathrm{co}} \mathcal{P}\left(E^{\mathrm{co}} ; T_{\text {eff }}\right)},
$$

from which we can compute the (relative) NMFs. Our fits will thus simply amount to determine the best value $T_{\text {eff }}$ for fixed values of $n$ in the aforementioned ranges reproducing the selected experimental data. 


\begin{tabular}{|cccc|}
\hline & $E_{\text {thr }}^{\mathcal{Q}}$ & $r_{\mathcal{Q}}$ & $\sigma_{\text {geo }}^{\mathcal{Q}}$ \\
\hline$\Upsilon(1 S)$ & $1100 \mathrm{MeV}$ & $0.14 \mathrm{fm}$ & $0.62 \mathrm{mb}$ \\
$\chi_{b}(1 P)$ & $670 \mathrm{MeV}$ & $0.22 \mathrm{fm}$ & $1.52 \mathrm{mb}$ \\
$\Upsilon(2 S)$ & $540 \mathrm{MeV}$ & $0.28 \mathrm{fm}$ & $2.46 \mathrm{mb}$ \\
$\chi_{b}(2 P)$ & $300 \mathrm{MeV}$ & $0.34 \mathrm{fm}$ & $3.63 \mathrm{mb}$ \\
$\Upsilon(3 S)$ & $200 \mathrm{MeV}$ & $0.40 \mathrm{fm}$ & $5.03 \mathrm{mb}$ \\
$\chi_{b}(3 P)$ & $50 \mathrm{MeV}$ & $0.55 \mathrm{fm}$ & $10.21 \mathrm{mb}$ \\
\hline
\end{tabular}

Table 1. Different fixed parameters used in our parametrisation of the $\Upsilon$-comover cross sections. The values of $E_{\mathrm{thr}}^{\mathcal{Q}}$ correspond to $m_{\mathrm{co}}=0$.

\section{Fitting the data}

In order to proceed with the fit, it is mandatory to take into account the feed-down (FD) contributions. The observed $\Upsilon(n S)$ yields indeed contain contributions from decays of heavier bottomonium states and, thus, the measured suppression can be affected by the dissociation of these states. This feed-down contribution to the $\Upsilon(1 S)$ state is usually asumed to be on the order of $50 \%$, according to the CDF measurements [36] at $p_{T}>8 \mathrm{GeV}$.

However, this assumption needs to be revisited, in particular for $p_{T}$-integrated results, following the more recent $\mathrm{LHCb}$ data extending to lower $p_{T}$ [37]. We refer to [38] for more details. We have reported the corresponding expected FD on the 3 first lines of table 2 . Note that for the $\Upsilon(3 S)$, the LHCb measurement is the only existing one and was done for $p_{T}>20 \mathrm{GeV}$.

Since these fractions remain partly extrapolated, one should consider them with some conservative uncertainties. We have thus varied the FD fractions used in our computations between two limiting cases: $80 \%$ of direct $\Upsilon(1 S)$ and $50 \%$ of direct $\Upsilon(3 S)$ - limiting case I - $60 \%$ of direct $\Upsilon(1 S)$ and $70 \%$ of direct $\Upsilon(3 S)$ - limiting case II - , leaving the other ones unchanged. All the corresponding values are collected on line 4-9 of table 2. This however induces changes which are not significant in view of the current experimental uncertainties.

As announced, we performed our fit on relative - minimum bias - NMFs. For $p \mathrm{~Pb}$ collisions, we have used the CMS [1] and ATLAS [9] data. For PbPb collisions, we have used the CMS data at $2.76 \mathrm{TeV}[11]$ and at $5.02 \mathrm{TeV}$ [19]. For both these $p \mathrm{~Pb}$ and $\mathrm{PbPb}$ cases, we performed the fit of $T_{\text {eff }}$ for different values of $n$ with both gluon or pion comovers. Our results are depicted on figure 1 . The resulting uncertainty on $T_{\text {eff }}$ is from the experimental uncertainty. Up to this uncertainty, all the combinations yield to the same couple $\left(n, T_{\text {eff }}\right)$ with $T_{\text {eff }}$ in the range 200 to $300 \mathrm{MeV}$ for our assumed range for $n$. Our fits are equally good with $\chi_{\text {d.o.f. }}^{2}$ ranging, for $p \mathrm{~Pb}$ data, from 1.0 to 1.4 and, for $\mathrm{PbPb}$ data, from 1.4 to 2.0.

Thus, we are confronted to the following quasi equiprobable possibilities:

Case I: the medium is of hadronic nature in $p \mathrm{~Pb}$ collisions, while it is gluonic in $\mathrm{PbPb}$ collisions.

Case II: both in $p \mathrm{~Pb}$ and $\mathrm{PbPb}$ collisions, the medium is made of hadrons, i.e. the comovers can be identified with pions. 


\begin{tabular}{|c|c|c|c|c|c|c|}
\hline & direct & $\chi_{b}(1 P) \mathrm{FD}$ & $\Upsilon(2 S) \mathrm{FD}$ & $\chi_{b}(2 P) \mathrm{FD}$ & $\Upsilon(3 S) \mathrm{FD}$ & $\chi_{b}(3 P) \mathrm{FD}$ \\
\hline \multicolumn{7}{|c|}{ From the } \\
\hline \multicolumn{7}{|c|}{ LHCb data } \\
\hline$\Upsilon(1 S)$ & $70 \%$ & $15 \%$ & $8 \%$ & $5 \%$ & $1 \%$ & $1 \%$ \\
\hline$\Upsilon(2 S)$ & $63 \%$ & - & - & $30 \%$ & $4 \%$ & $3 \%$ \\
\hline$\Upsilon(3 S)$ & $60 \%$ & - & - & - & - & $40 \%$ \\
\hline \multicolumn{7}{|l|}{ Limiting } \\
\hline \multicolumn{7}{|l|}{ Case I } \\
\hline$\Upsilon(1 S)$ & $80 \%$ & $10 \%$ & $5.3 \%$ & $3.3 \%$ & $0.7 \%$ & $0.7 \%$ \\
\hline$\Upsilon(2 S)$ & $63 \%$ & - & - & $30 \%$ & $4 \%$ & $3 \%$ \\
\hline$\Upsilon(3 S)$ & $50 \%$ & - & - & - & - & $50 \%$ \\
\hline \multicolumn{7}{|l|}{ Limiting } \\
\hline \multicolumn{7}{|l|}{ Case II } \\
\hline$\Upsilon(1 S)$ & $60 \%$ & $20 \%$ & $10.7 \%$ & $6.7 \%$ & $1.3 \%$ & $1.3 \%$ \\
\hline$\Upsilon(2 S)$ & $63 \%$ & - & - & $30 \%$ & $4 \%$ & $3 \%$ \\
\hline$\Upsilon(3 S)$ & $70 \%$ & - & - & - & - & $30 \%$ \\
\hline
\end{tabular}

Table 2. Expected $\Upsilon$ FD contributions.

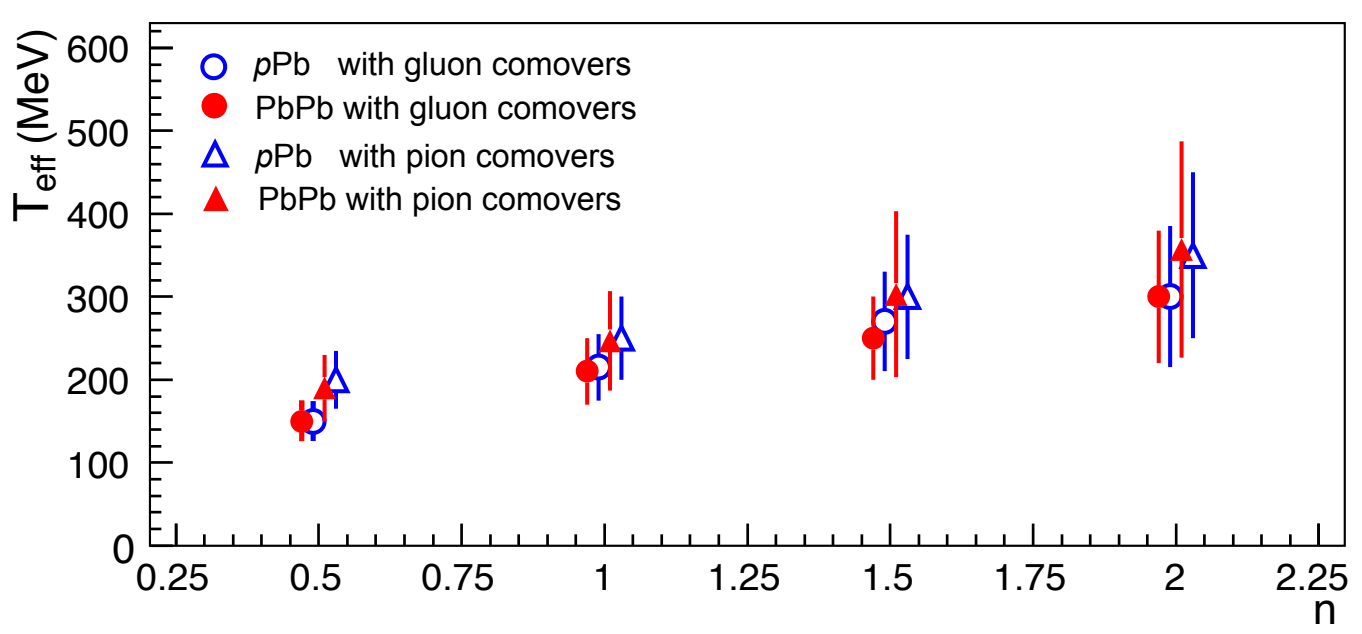

Figure 1. Resulting $T_{\text {eff }}$ for pion (triangles) or gluon (circles) comovers from our fits to $p \mathrm{~Pb}$ (empty blue) and $\mathrm{PbPb}$ (filled red) data for different $n$ from 0.5 to 2. [The points have been horizontally shifted for readibility.] 
Case III: both in $p \mathrm{~Pb}$ and $\mathrm{PbPb}$ collisions, the medium is made of partons, i.e. the comovers can be identified with gluons.

Case IV: the medium is of gluonic nature in $p \mathrm{~Pb}$ collisions, while it is hadronic in $\mathrm{PbPb}$ collisions.

Case I is the most common expectation. The relevant d.o.f. are hadrons in $p \mathrm{~Pb}$ collisions where the QGP is not produced whereas the gluons become relevant in the hotter $\mathrm{PbPb}$ environment with the presence of QGP. Case II is the usual interpretation of historical CIM studies for which the gluon d.o.f. do not appear to be relevant. At SPS energies, it is a reasonable assumption. At the LHC, it is more thought-provoking, yet compatible with the observed bottomonium suppression at the LHC. It can also be understood in the sense that the melting temperature of the $\Upsilon(1 S)$ and $\Upsilon(2 S)$ is too high to be observed and the $\Upsilon(3 S)$ is fragile enough to be entirely broken by hadrons. Case III amounts to say that gluons are the relevant d.o.f. to account for bottomonium suppression in both $p \mathrm{~Pb}$ and in $\mathrm{PbPb}$ collisions. One could thus say that a QGP-like medium is formed following $p \mathrm{~Pb}$ collisions at LHC energies. Case IV is admittedly an unexpected situation.

In what follows, our results for the NMFs will be shown for $n=1$ and $T_{\text {eff }}=$ $250 \pm 50 \mathrm{MeV}$. Choosing different couples of $n$ and $T_{\text {eff }}$ yield to very similar results since the variation of $n$ is compensated by that of $T_{\text {eff }}$. Showing them for each of the tested hypothesis would not bring in any additional information in view of the current experimental uncertainties and of the uncertainties from the nuclear PDFs - in the case of the absolute NMFs.

As what regards their specific values, for an exponent $n=1,\left\langle\sigma^{\mathrm{co}-\Upsilon(1 \mathrm{~S})}\right\rangle$ is $0.02_{-0.01}^{+0.02} \mathrm{mb}$ for the most tightly bound state $\Upsilon(1 S)$, compatible with no suppression of the direct $\Upsilon(1 S)$, while $\left\langle\sigma^{\text {co- }-\chi_{\mathrm{b}}(3 \mathrm{P})}\right\rangle=9.2_{-1.4}^{+1.0} \mathrm{mb}$ for the loosely bound $\chi_{b}(3 P)$ states in the hadronic case. The quoted uncertainty comes from that on the temperature, i.e. $T_{\text {eff }}=250 \pm 50 \mathrm{MeV}$ for $n=1$, which is generated by the experimental uncertainties via the $\chi^{2}$ minimisation.

Looking at these cross sections allows us to better understand the small impact of considering gluon or pion comovers. In fact, the mass effects only matter for $\chi_{b}(3 P)$ states altering their interaction cross section by $10 \%$. They however does not induce any visible difference. Indeed, for such large cross sections, the obtained suppression is already maximal for minimum bias collisions.

\section{Relative NMFs}

The resulting relative NMFs of the excited bottomonium states to their ground state in $p \mathrm{~Pb}$ collisions at $5.02 \mathrm{TeV}$ are presented in table 3 along with the CMS [1] and ATLAS [9] experimental data. We note that the central values of the data tend to indicate a slightly stronger suppression that our results. We however recall that so far no other model could explain this relative suppression in $p \mathrm{~Pb}$ collisions.

In $\mathrm{PbPb}$ collisions, besides the minimum-bias values which we used in our fits, CMS reported on the centrality dependence of the relative suppression of $\Upsilon(n S)$ at 2.76 and 


\begin{tabular}{|lcc|}
\hline & CIM fit & Experimental values \\
\hline & $-1.93<y<1.93$ & CMS data \\
$\Upsilon(2 S) / \Upsilon(1 S)$ & $0.91 \pm 0.03$ & $0.83 \pm 0.05$ (stat.) \pm 0.05 (syst.) \\
$\Upsilon(3 S) / \Upsilon(1 S)$ & $0.72 \pm 0.02$ & $0.71 \pm 0.08$ (stat.) \pm 0.09 (syst.) \\
& $-2.0<y<1.5$ & ATLAS data \\
$\Upsilon(2 S) / \Upsilon(1 S)$ & $0.90 \pm 0.03$ & $0.76 \pm 0.07$ (stat.) \pm 0.05 (syst.) \\
$\Upsilon(3 S) / \Upsilon(1 S)$ & $0.71 \pm 0.02$ & $0.64 \pm 0.14$ (stat.) \pm 0.06 (syst.) \\
\hline
\end{tabular}

Table 3. Measured and fit $R_{p \mathrm{~Pb}}^{\Upsilon(m S) / \Upsilon(n S)}$ at $5.02 \mathrm{TeV}$.

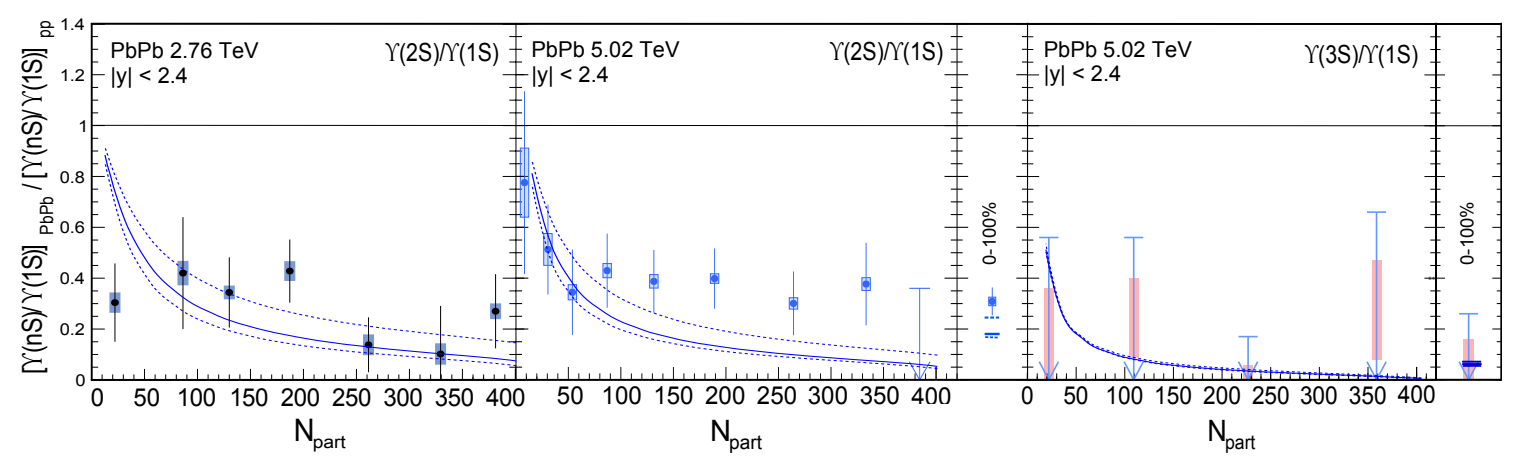

Figure 2. The double ratio $\left(R_{\mathrm{PbPb}}^{\Upsilon(m S) / \Upsilon(n S)}\right)$ for $\Upsilon(2 S)$ over $\Upsilon(1 S)$ at 2.76 and $5.02 \mathrm{TeV}$ and $\Upsilon(3 S)$ over $\Upsilon(1 S)$ at $5.02 \mathrm{TeV}$ as a function of $N_{\text {part }}$ obtained from the CIM with the CMS data at $2.76 \mathrm{TeV}[11]$ and $5.02 \mathrm{TeV}[19]$. The dashed line depicts the uncertainty from the fit of $\sigma^{\mathrm{co}-\Upsilon}$.

5.02 TeV [11, 19]. Figure 2 shows our results along with the CMS points. The agreement is very good at $2.76 \mathrm{TeV}$, a bit less for $\Upsilon(2 S) / \Upsilon(1 S)$ at $5.02 \mathrm{TeV}$.

\section{Absolute NMFs}

Having fixed the parameters of our approach with the relative suppression measurements, we can now address the absolute suppression of each measured states. However, when addressing the absolute $\Upsilon$ suppression, other nuclear effects, which cancel in the double ratio of the excited-to-ground state suppression, do not cancel anymore. At LHC energies, the main one seems to be [39] the nuclear modification of PDFs. It is easily accounted for by using available global $\mathrm{nPDF}$ fits with uncertainties $[2,4,5,40]$.

In particular, we used nCTEQ15 which describes very well the suppression of open charm in $p \mathrm{~Pb}$ collisions at the LHC $[39,43]$. We also note that the central value of the nCTEQ15 fit is compatible with the one of EPS09LO [5] previously used in [28].

Let us first start with the $\Upsilon(1 S)$ case in $p \mathrm{~Pb}$ collisions. Figure 3 shows $R_{p \mathrm{~Pb}}^{\Upsilon(1 S)}$ vs rapidity at $\sqrt{s}=5.02 \mathrm{TeV}$ compared to the available experimental data $[9,41,42]$ from ALICE, ATLAS and LHCb. The agreement is overall very good and the additional effect of the CIM is to damp down the antishadowing peak in the backward rapidity region which brings the theory closer to the central value of ALICE. 


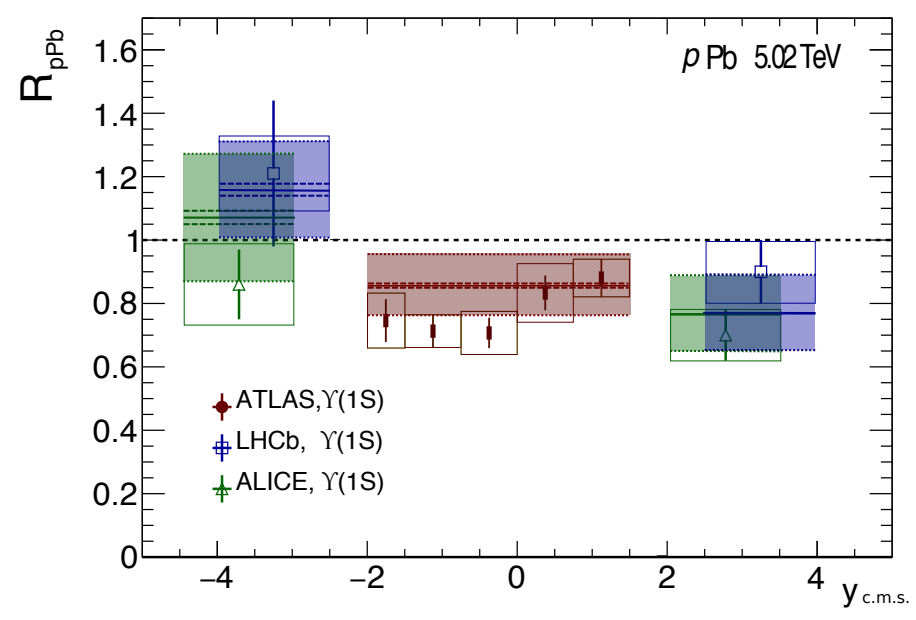

Figure 3. The $R_{p \mathrm{~Pb}}^{\Upsilon(1 S)}$ vs rapidity compared to the LHC data [9, 41, 42]. The uncertainty from the $\sigma^{\text {co- } \Upsilon}$ fit (dashed line) and from the nCTEQ15 shadowing (dotted line along with the colored band) are shown separately.
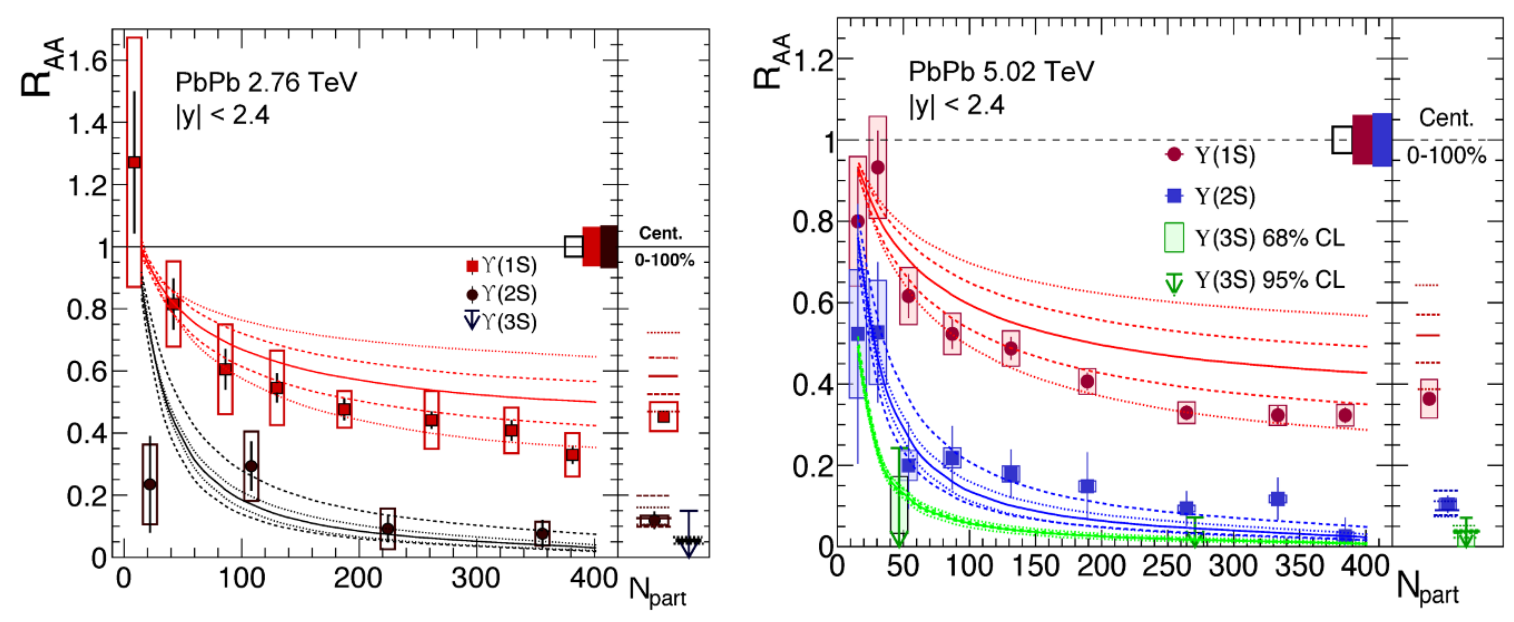

Figure 4. $R_{\mathrm{PbPb}}^{\Upsilon(n S)}$ vs. $N_{\text {part }}$ from the CIM with the CMS data at $2.76 \mathrm{TeV}$ [44] and $5.02 \mathrm{TeV}$ [45]. The uncertainty from the fit of $\sigma^{\mathrm{co}-\Upsilon}$ (dashed line) and from the nCTEQ15 shadowing (dotted line) are shown separately.

We then compare our results to $\mathrm{PbPb}$ data whose centrality dependence has also been measured. To address this dependence, we parameterised the impact-parameter dependence of the nPDF as in [28]. Our results for the $3 \Upsilon$ states at 2.76 (5.02) TeV are shown in figure 4 left (right) with the CMS data [44, 45]. A good agreement is obtained in the 3 cases with the same parameters used to reproduce the relative suppression.

\section{Conclusions}

In this work, we have addressed the puzzle of the relative suppression of the excited bottomonium states as compared to their ground state in $p \mathrm{~Pb}$ collisions. In the absence of any other explanation, we have assumed that the reinteraction with comovers explains it 
all. This motivated us to revisit the CIM and to propose a generic formula for all the bottomonium states interpolating from the absence of interaction at threshold up to the geometrical one for increasing comover-quarkonium relative momenta in the transverse plane. Taking into account the energy distribution of the comovers with a Bose-Einstein distribution, we could further study the impact of considering either massive pion or massless gluon comovers and investigate an effective temperature of the comovers as probed by the quarkonia. This allowed us to fit the CMS and ATLAS $p \mathrm{~Pb}$ double ratios with only 2 parameters accounting for all the comover-bottomonium interaction cross sections. With the same setup, an independent fit of the corresponding $\mathrm{PbPb}$ CMS data yielded similar fit parameter values, thus hinting at a similar momentum distribution of these comovers in the environment created by $p A$ and $A A$ collisions. This is admittedly an unexpected and very interesting observation.

We further backed up our investigations by noting that our approach correctly predicted the absolute $\Upsilon$ suppression in both $p \mathrm{~Pb}$ and $\mathrm{PbPb}$ collisions when combined with the nCTEQ15 shadowing without the need to invoke any other phenomena.

\section{Acknowledgments}

We would like to thank Francois Arleo, Alfons Capella, Olivier Drapier and Frederic Fleuret for stimulating and useful discussions. E.G.F. thanks Laboratoire LePrince-Ringuet de l'Ecole Polytechnique and l'Université Paris-Saclay for their hospitality and financial support in the framework of Jean D'Alembert fellowship during the completion of this work, and to the Ministerio de Ciencia e Innovacion of Spain under project FPA2014-58293C2-1-P and Unidad de Excelencia María de Maetzu under project MDM-2016-0692 for financial support. The work of J.P.L. is supported in part by the French CNRS via the grant PICS-07920 "Excitonium".

Open Access. This article is distributed under the terms of the Creative Commons Attribution License (CC-BY 4.0), which permits any use, distribution and reproduction in any medium, provided the original author(s) and source are credited.

\section{References}

[1] CMS collaboration, Event activity dependence of $Y(n S)$ production in $\sqrt{s_{N N}}=5.02 \mathrm{TeV}$ $p P b$ and $\sqrt{s}=2.76 \mathrm{TeV}$ pp collisions, JHEP 04 (2014) 103 [arXiv:1312.6300] [INSPIRE].

[2] K. Kovarik et al., nCTEQ15 - Global analysis of nuclear parton distributions with uncertainties in the CTEQ framework, Phys. Rev. D 93 (2016) 085037 [arXiv:1509.00792] [INSPIRE].

[3] J.F. Owens, A. Accardi and W. Melnitchouk, Global parton distributions with nuclear and finite- $Q^{2}$ corrections, Phys. Rev. D 87 (2013) 094012 [arXiv: 1212.1702] [inSPIRE].

[4] D. de Florian, R. Sassot, P. Zurita and M. Stratmann, Global Analysis of Nuclear Parton Distributions, Phys. Rev. D 85 (2012) 074028 [arXiv:1112.6324] [InSPIRE].

[5] K.J. Eskola, H. Paukkunen and C.A. Salgado, EPSO9: A New Generation of NLO and LO Nuclear Parton Distribution Functions, JHEP 04 (2009) 065 [arXiv: 0902.4154] [INSPIRE]. 
[6] M. Hirai, S. Kumano and T.H. Nagai, Determination of nuclear parton distribution functions and their uncertainties in next-to-leading order, Phys. Rev. C 76 (2007) 065207 [arXiv: 0709.3038] [INSPIRE].

[7] F. Arleo and S. Peigné, Quarkonium suppression in heavy-ion collisions from coherent energy loss in cold nuclear matter, JHEP 10 (2014) 073 [arXiv:1407.5054] [INSPIRE].

[8] E.G. Ferreiro, F. Fleuret, J.P. Lansberg, N. Matagne and A. Rakotozafindrabe, $\Upsilon$ production in $p(d) A$ collisions at RHIC and the LHC, Eur. Phys. J. C 73 (2013) 2427 [arXiv: 1110.5047] [INSPIRE].

[9] ATLAS collaboration, Measurement of quarkonium production in proton-lead and proton-proton collisions at $5.02 \mathrm{TeV}$ with the ATLAS detector, Eur. Phys. J. C 78 (2018) 171 [arXiv: 1709. 03089] [INSPIRE].

[10] CMS collaboration, Indications of suppression of excited $\Upsilon$ states in PbPb collisions at $\sqrt{S_{N N}}=2.76 \mathrm{TeV}$, Phys. Rev. Lett. 107 (2011) 052302 [arXiv:1105.4894] [INSPIRE].

[11] CMS collaboration, Observation of sequential $\Upsilon$ suppression in PbPb collisions, Phys. Rev. Lett. 109 (2012) 222301 [Erratum ibid. 120 (2018) 199903] [arXiv: 1208. 2826] [INSPIRE].

[12] J.P. Lansberg, Theory status of quarkonium production in proton-nucleus collisions, J. Phys. Conf. Ser. 668 (2016) 012019 [arXiv:1510.01818] [InSPIRE].

[13] E.G. Ferreiro, F. Fleuret, J.P. Lansberg and A. Rakotozafindrabe, Cold nuclear matter effects on $J / \psi$ production: Intrinsic and extrinsic transverse momentum effects, Phys. Lett. B 680 (2009) 50 [arXiv:0809.4684] [InSPIRE].

[14] E.G. Ferreiro, F. Fleuret, J.P. Lansberg and A. Rakotozafindrabe, Centrality, Rapidity and Transverse-Momentum Dependence of Cold Nuclear Matter Effects on $J / \psi$ Production in $d A u, C u C u$ and AuAu Collisions at $\sqrt{s_{N N}}=200 \mathrm{GeV}$, Phys. Rev. C 81 (2010) 064911 [arXiv: 0912.4498] [INSPIRE].

[15] R. Vogt, Cold Nuclear Matter Effects on $J / \psi$ and $\Upsilon$ Production at the LHC, Phys. Rev. C 81 (2010) 044903 [arXiv: 1003.3497] [INSPIRE].

[16] E.G. Ferreiro, F. Fleuret, J.P. Lansberg and A. Rakotozafindrabe, Impact of the Nuclear Modification of the Gluon Densities on $J / \psi$ production in $p P b$ collisions at $\sqrt{s_{N N}}=5 \mathrm{TeV}$, Phys. Rev. C 88 (2013) 047901 [arXiv:1305.4569] [InSPIRE].

[17] J.L. Albacete et al., Predictions for $p+P b$ Collisions at $\sqrt{s_{N N}}=5$ TeV: Comparison with Data, Int. J. Mod. Phys. E 25 (2016) 1630005 [arXiv:1605.09479] [inSPIRE].

[18] J.L. Albacete et al., Predictions for Cold Nuclear Matter Effects in $p+P b$ Collisions at $\sqrt{s_{N N}}=8.16 \mathrm{TeV}$, Nucl. Phys. A 972 (2018) 18 [arXiv:1707.09973] [inSPIRE].

[19] CMS collaboration, Suppression of Excited $\Upsilon$ States Relative to the Ground State in $\mathrm{Pb}-\mathrm{Pb}$ Collisions at $\sqrt{s_{N N}}=5.02$ TeV, Phys. Rev. Lett. 120 (2018) 142301 [arXiv:1706. 05984] [INSPIRE].

[20] A. Capella, A. Kaidalov, A. Kouider Akil and C. Gerschel, $J / \psi$ and $\psi^{\prime}$ suppression in heavy ion collisions, Phys. Lett. B 393 (1997) 431 [hep-ph/9607265] [InSPIRE].

[21] N. Armesto and A. Capella, A quantitative reanalysis of charmonium suppression in nuclear collisions, Phys. Lett. B 430 (1998) 23 [hep-ph/9705275] [INSPIRE].

[22] N. Armesto, A. Capella and E.G. Ferreiro, Charmonium suppression in lead-lead collisions: Is there a break in the $J / \psi$ cross-section?, Phys. Rev. C 59 (1999) 395 [hep-ph/9807258] [INSPIRE]. 
[23] A. Capella, E.G. Ferreiro and A.B. Kaidalov, Nonsaturation of the $J / \psi$ suppression at large transverse energy in the comovers approach, Phys. Rev. Lett. 85 (2000) 2080 [hep-ph/0002300] [INSPIRE].

[24] A. Capella and E.G. Ferreiro, $J / \psi$ suppression at $\sqrt{s}=200 \mathrm{GeV}$ in the comovers interaction model, Eur. Phys. J. C 42 (2005) 419 [hep-ph/0505032] [INSPIRE].

[25] A. Capella and E.G. Ferreiro, $J / \psi$ suppression and the decrease of nuclear absorption with increasing energy, Phys. Rev. C 76 (2007) 064906 [hep-ph/0610313] [INSPIRE].

[26] A. Capella, L. Bravina, E.G. Ferreiro, A.B. Kaidalov, K. Tywoniuk and E. Zabrodin, Charmonium dissociation and recombination at RHIC and LHC, Eur. Phys. J. C 58 (2008) 437 [arXiv: 0712.4331] [INSPIRE].

[27] E.G. Ferreiro, Charmonium dissociation and recombination at LHC: Revisiting comovers, Phys. Lett. B 731 (2014) 57 [arXiv:1210.3209] [inSPIRE].

[28] E.G. Ferreiro, Excited charmonium suppression in proton-nucleus collisions as a consequence of comovers, Phys. Lett. B $\mathbf{7 4 9}$ (2015) 98 [arXiv:1411.0549] [InSPIRE].

[29] A. Capella and E.G. Ferreiro, Charged multiplicities in pp and AA collisions at LHC, Eur. Phys. J. C 72 (2012) 1936 [arXiv:1110.6839] [InSPIRE].

[30] H. Satz, Colour deconfinement and quarkonium binding, J. Phys. G 32 (2006) R25 [hep-ph/0512217] [INSPIRE].

[31] G. Bhanot and M.E. Peskin, Short Distance Analysis for Heavy Quark Systems. 2. Applications, Nucl. Phys. B 156 (1979) 391 [inSPIRE].

[32] D. Kharzeev and H. Satz, Quarkonium interactions in hadronic matter, Phys. Lett. B 334 (1994) 155 [hep-ph/9405414] [INSPIRE].

[33] R. Rapp, D. Blaschke and P. Crochet, Charmonium and bottomonium production in heavy-ion collisions, Prog. Part. Nucl. Phys. 65 (2010) 209 [arXiv:0807.2470] [inSPIRE].

[34] K. Martins, D. Blaschke and E. Quack, Quark exchange model for charmonium dissociation in hot hadronic matter, Phys. Rev. C 51 (1995) 2723 [hep-ph/9411302] [INSPIRE].

[35] T. Song and S.H. Lee, Quarkonium-hadron interactions in perturbative QCD, Phys. Rev. D 72 (2005) 034002 [hep-ph/0501252] [INSPIRE].

[36] CDF collaboration, T. Affolder et al., Production of $\Upsilon(1 S)$ mesons from $\chi_{b}$ decays in $p \bar{p}$ collisions at $\sqrt{s}=1.8$ TeV, Phys. Rev. Lett. 84 (2000) 2094 [hep-ex/9910025] [INSPIRE].

[37] LHCb collaboration, Study of $\chi_{b}$ meson production in $p p$ collisions at $\sqrt{s}=7$ and $8 \mathrm{TeV}$ and observation of the decay $\chi_{b}(3 P) \rightarrow \Upsilon(3 S) \gamma$, Eur. Phys. J. C 74 (2014) 3092 [arXiv: 1407.7734] [INSPIRE].

[38] A. Andronic et al., Heavy-flavour and quarkonium production in the LHC era: from proton-proton to heavy-ion collisions, Eur. Phys. J. C 76 (2016) 107 [arXiv:1506.03981] [INSPIRE].

[39] A. Kusina, J.-P. Lansberg, I. Schienbein and H.-S. Shao, Gluon Shadowing in Heavy-Flavor Production at the LHC, Phys. Rev. Lett. 121 (2018) 052004 [arXiv:1712.07024] [inSPIRE].

[40] K.J. Eskola, P. Paakkinen, H. Paukkunen and C.A. Salgado, EPPS16: Nuclear parton distributions with LHC data, Eur. Phys. J. C 77 (2017) 163 [arXiv:1612.05741] [INSPIRE].

[41] LHCb collaboration, Study of $\Upsilon$ production and cold nuclear matter effects in $p P b$ collisions at $\sqrt{s_{N N}}=5 \mathrm{TeV}$, JHEP 07 (2014) 094 [arXiv: 1405.5152] [INSPIRE]. 
[42] ALICE collaboration, Production of inclusive $\Upsilon(1 S)$ and $\Upsilon(2 S)$ in $p$-Pb collisions at $\sqrt{s_{N N}}=5.02 \mathrm{TeV}$, Phys. Lett. B 740 (2015) 105 [arXiv: 1410.2234] [INSPIRE].

[43] J.-P. Lansberg and H.-S. Shao, Towards an automated tool to evaluate the impact of the nuclear modification of the gluon density on quarkonium, $D$ and $B$ meson production in proton-nucleus collisions, Eur. Phys. J. C 77 (2017) 1 [arXiv:1610.05382] [inSPIRE].

[44] CMS collaboration, Suppression of $\Upsilon(1 S), \Upsilon(2 S)$ and $\Upsilon(3 S)$ production in $P b P b$ collisions at $\sqrt{s_{\mathrm{NN}}}=2.76 \mathrm{TeV}$, Phys. Lett. B 770 (2017) 357 [arXiv:1611.01510] [INSPIRE].

[45] CMS collaboration, Measurement of Nuclear Modification Factors of $\Upsilon(n S)$ Mesons in $\mathrm{PbPb}$ Collisions at $\sqrt{s_{N N}}=5.02 \mathrm{TeV}$, CMS-PAS-HIN-16-023 (2017) [INSPIRE]. 